Letters to the Editor

\section{Evidence of Nosocomial Stenotrophomonas maltophilia Cross- Infection in a Neonatology Unit Analyzed by Three Molecular Typing Methods}

\section{To the Editor:}

I read with interest the paper by García de Viedma et $\mathrm{al}^{1}$ on Stenotrophomonas maltophilia. Although not the first evidence of nosocomial cross-transmission of this microorganism, the authors' use of molecular methods was thorough and contributed to what is known about this important organism. We used the molecular techniques contour-clamped homogeneous electric-field gel electrophoresis ${ }^{2}$ and arbitrarily primed polymerase chain reaction ${ }^{3}$ to compare 64 hospital endemic and neonatal intensive care unit epidemic isolates of $S \mathrm{mal}$ tophilia. Additionally, we conducted a retrospective chart review to determine risk factors. ${ }^{4}$ Our results showed that, while nosocomial transmission does occur, most isolates are acquired independently as a consequence of risk factors such as prior antibiotic therapy.

Why is it important to know how patients become infected with this organism? So that we can make decisions on how to protect them. What should we do, for example, when two isolates of $S$ maltophilia are found on the same unit? If we assume them to be cross-transmitted, we easily become immersed in a finger-pointing war to see who can be blamed for breaking isolation technique. The doctors blame the nurses, the nurses blame the doctors, and everyone blames housekeeping. If we assume isolates to arise from diverse environmental sources, we fail to isolate properly. What is the infection control clinician to do? Should resources be utilized at all? When molecular data are available, these questions are relatively easy to answer. Most clinicians, however, must make these decisions without the assistance of this typing technology. Although rarely seen in a modern research paper, the old standby, antibiotic susceptibility patterns, can be useful, even with a multiply resistant organism. We showed antibiograms to have a Simpson's Discriminatory Index of 0.87 when applied to 56 isolates of $S$ maltophilia. ${ }^{2}$ Although analyzing variability in antibiograms becomes extremely complicated when multiple strains are involved, a single pair can often be analyzed efficiently and economically. The data may help a bedside clinician motivate others to expend resources constructively to prevent further infections.

\section{REFERENCES}

1. Garcia de Viedma D, Marin M, Cercenado $E$ Alonso R, Rodriguez-Creixems M, Bouza E. Evidence of nosocomial Stenotrophomonas maltophilia cross-infection in a neonatology unit analyzed by three molecular typing methods. Infect Control Hosp Epidemiol 1999;20:816-820.

2. VanCouwenberghe CJ, Cohen S. Analysis of epidemic and endemic isolates of Xanthomonas maltophilia by contourclamped homogeneous electric field gel electrophoresis. Infect Control Hosp Epidemiol 1994;15:691-696.

3. VanCouwenberghe CJ, Cohen SH, Tang YJ, Gumerlock PH, Silva IS. Genomic fingerprinting of epidemic and endemic strains of Stenotrophomonas maltophilia (formerly Xanthomonas maltophilia) by arbitrarily primed PCR. J Clin Microbiol 1995;33:12891291.

4. VanCouwenberghe CJ, Farver TB, Cohen $\mathrm{SH}$. Risk factors associated with isolation of Stenotrophomonas (Xanthomonas) maltophil$i a$ in clinical specimens. Infect Control Hosp Epidemiol 1997;18:316-321.

Carolyn VanCouwenberghe, RN, PhD California State University-Sacramento University of California Davis Sacramento, California

\section{The authors reply.}

We appreciate Dr VanCouwenberghe's interest in our article. Her group has obtained solid data about Stenotrophomonas maltophilia noso- comial transmission based on molecular analysis. Additionally, they have made some interesting observations, such as the hypothesis that some cases of nosocomial infections by this microorganism could be due to community-acquired isolates after the selective pressure exerted by antibiotic therapy. This would explain the high genetic variability frequently associated with nosocomial $S$ maltophilia isolates.

Regarding her observation that ours is not the first evidence of nosocomial cross-transmission due to this microorganism, we would like to make some comments. Several other authors before us have found molecular similarities among nosocomial $S$ maltophilia isolates. Nevertheless, to our knowledge, none of them have fulfilled the epidemiological conditions that allowed us to assign a role to cross-transmission (meaning patientto-patient transmission) in the nosocomial outbreak.

The article by VanCouwenberghe et al discusses the notion that the similarities found among the molecular fingerprints of a group of isolates indicate that this microorganism can be transmitted from patient to patient. They support this conclusion because of the chronological overlap found in patients with isolates sharing fingerprints. It is worth noting that, in their study, all $S$ maltophilia isolates were obtained after several days of hospital stay for all patients. In our opinion, this leaves two possible causes for the outbreak, either patient-to-patient transmission or the participation of a unique environmental source.

On the contrary, in our study, the first isolate in the outbreak corresponded to a neonate, transferred from another institution, with an $S$ maltophilia isolate drawn from a culture taken on the very day of admission to the neonatology unit. This strongly rules out the possibility of an environmental source for this neonatal infection. Additionally, all but one of the isolates in the outbreak clustered at $96 \%$ similarity 
with the isolate imported by this neonate from the other institution. These two observations together highly minimize the role of a unique environmental source and lead us to consider our study as the first evidence of patient-to-patient nosocomial cross-transmission by $S$ maltophilia.

We share Dr. VanCouwenberghe's opinion about the importance of defining the nosocomial transmission dynamics of this microorganism. The precise definition of either environmental sources or cross-transmission as the causes of nosocomial infections is essential in order to adopt specific strategies in the management of patients and thus efficiently contain outbreaks.

With regard to the proposal of using antibiotic susceptibility patterns to type this microorganism, in our case this approach was not possible, as all isolates were multiresistant and susceptible only to trimethoprim-sulfamethoxazole. Furthermore, in our institution, most of the $S$ maltophilia isolates (70\%) obtained in 1999 were susceptible to only trimethoprim-sulfamethoxazole (50\%) or to aminoglycosides and quinolones (20\%). This would limit seriously, in our context, the usefulness of applying susceptibility patterns for typing purposes.

We consider that, today, molecular typing is a requirement for efficient management of nosocomial infections, and it should no longer be considered as a luxury tool restricted to refined epidemiological analysis. To fill the conceptual gap between these two realities, efforts should be made to increase personnel expertise in molecular techniques in the microbiology wards and to supply them with adequate resources. The reduction in expenses derived from more efficient management of nosocomial infections would easily finance "typing units" to provide immediate assistance to the bedside clinician.

Dario García de Viedma, $\mathbf{P h D}$ Mercedes Marín, PhD

Emilia Cercenado, PharmD Roberto Alonso, PhD

Marta Rodriguez Créixems, $\mathrm{MD}, \mathrm{PhD}$ Emilio Bouza, MD, PhD

Hospital General Universitario Gregorio Marañón

Madrid, Spain
Hospital Characteristics Associated With Colonization of Water Systems by Legionella and Risk of Nosocomial Legionnaires' Disease: A Cohort Study of 15 Hospitals

\section{To the Editor:}

We wish to comment on the important article authored by Kool et al from the Centers for Disease Control and Prevention (CDC). ${ }^{1}$ Kool et al found that the number of cases of hospital-acquired legionnaires' disease in San Antonio hospitals correlated better with the proportion of water sites positive for Legionella than with concentration of Legionella in water samples. That is, quantitation of Legionella at individual distal water sites did not correlate well with the presence of hospital-acquired legionnaires' disease. We had already documented this phenomenon, ${ }^{2}$ as pointed out by Kool et al. This information is reflected in the Allegheny County Health Department (ACHD) guidelines mandating routine environmental cultures for Legionella in Pittsburgh hospitals; the proportion of sites positive, not quantitation at distal sites, is the parameter used for decision making. ${ }^{3}$ In contrast, the CDC guidelines recommend obtaining environmental cultures after only one or two cases of hospital-acquired legionnaires' disease are discovered. ${ }^{4}$

Kool et al noted that it may seem counterintuitive that Legionella bacterial concentrations would not be an important determination of risk of transmission. The major reason why bacterial concentrations are not predictive is that Legionella is not concentrated in water but is harbored in the biofilm consisting of sediment and detritus at each distal water outlet. 5,6 Thus, sampling itself will affect the biofilm, and repeated samplings over time may well dislodge the biofilm present in any distal water fixture, thus decreasing the quantitative count. We have also shown that the concentration of Legionella in a water sample can be significantly lower (even negative) than that recovered by a swab (biofilm) sample. ${ }^{5}$

The CDC .errs in claiming that the ACHD guidelines recommend that disinfection measures be implemented when the percentage of positive sites exceeds $30 \%$. Actually, the ACHD guidelines recommend only that disinfection be considered at that level, but the guidelines do mandate that Legionella testing be made available in hospitals with a contaminated water supply. The ACHD guidelines are much less draconian than the CDC guidelines. ${ }^{4}$ For example, the $\mathrm{CDC}$ guidelines recommend that the percentage of positive sites and quantitative counts be reduced to zero, which is extremely difficult to attain, especially with hyperchlorination. Since there is some debate regarding the level of site positivity that should trigger disinfection options, it would be meaningful to know whether Kool et al found a specific percentage of positive sites above which disease was more likely to be detected.

It is well recognized that the diagnosis of legionnaires' disease cannot be made on clinical grounds alone and that specialized laboratory tests are necessary, as is well shown in the article by Kool et al. The authors point out that numerous cases may have been missed, because Legionella testing was not routinely applied to all patients with hospital-acquired pneumonia. The authors do not mention the mortality for the legionnaires' disease cases in San Antonio.

We cannot help but point out that, had the ACHD guidelines been implemented in the 12 San Antonio hospitals, all of these hospitals would have adopted in-house Legionella laboratory testing many years earlier. Cases would have been uncovered earlier if urinary antigen testing and culture on selective media were applied to patients with hospitalacquired pneumonia. Initiation of earlier treatment would have minimized the mortality. Disinfection measures could be considered, in order to prevent more cases from occurring. The proactive ACHD approach versus the reactive $\mathrm{CDC}$ approach actually can be assessed prospectively in San Antonio.

Although Legionella was found in 11 hospitals, only 5 hospitals experienced hospital-acquired legionnaires' disease. We predict that the remaining 6 hospitals may well have undiagnosed hospital-acquired legionnaires' disease, if the proportion of site positivity is high. We recommend that the microbiology laboratories of these 6 hospitals perform urinary antigen 